\title{
Microscopic density matrix model for optical gain of terahertz quantum cascade lasers: Many-body, nonparabolicity, and resonant tunneling effects
}

\author{
Tao Liu, ${ }^{1,2}$ Kenneth E. Lee, ${ }^{3}$ and Qi Jie Wang ${ }^{1,2,4, *}$ \\ ${ }^{1}$ School of Electrical \& Electronic Engineering, Nanyang Technological University, 50 Nanyang Ave., Singapore, 639798 \\ ${ }^{2}$ CINTRA CNRS/NTU/THALES, UMI 3288, Research Techno Plaza, 50 Nanyang Drive, Border X Block, Level 6, Singapore, 637553 \\ ${ }^{3}$ Temasek Laboratories, Nanyang Technological University, 50 Nanyang Ave, Singapore, 639798 \\ ${ }^{4}$ School of Physical and Mathematical Sciences, Nanyang Technological University, Singapore, 637371
}

(Received 10 August 2012; revised manuscript received 7 November 2012; published 11 December 2012)

\begin{abstract}
Intersubband semiconductor-Bloch equations are investigated by incorporating many-body Coulomb interaction, nonparabolicity, and coherence of resonant tunneling transport in a quantitative way based on the density matrix theory. The calculations demonstrate the importance of these parameters on optical properties, especially the optical gain spectrum, of terahertz (THz) quantum cascade lasers (QCLs). The results show that the lasing frequency at gain peak calculated by the proposed microscopic density matrix model is closer to the experimentally measured result, compared with that calculated by the existing macroscopic density matrix model. Specifically, both the many-body interaction and nonparabolicity effects red-shift the gain spectrum and reduce the gain peak. In addition, as the injection-coupling strength increases, the gain peak value is enhanced and the spectrum is slightly broadened, while an increase of the extraction-coupling strength reduces the gain peak value and broadens the gain spectrum. The dependence of optical gain of THz QCLs on device parameters such as external electrical bias, dephasing rate, doping density, and temperature is also systematically studied in details. This model provides a more comprehensive picture of the optical properties of THz QCLs from a microscopic point of view and potentially enables a more accurate and faster prediction and calculation of the device performance, e.g., gain spectra, current-voltage characteristics, optical output powers, and nonlinear amplitude-phase coupling.
\end{abstract}

DOI: 10.1103/PhysRevB.86.235306

PACS number(s): 42.55.Ah, 73.63.-b, 73.40.Gk, 78.67.-n

\section{INTRODUCTION}

Since the first demonstration in 1994, quantum cascade lasers (QCLs) have become important coherent mid-infrared $(\mathrm{Mid}-\mathrm{IR})^{1}$ and terahertz $(\mathrm{THz})^{2}$ radiation sources. Roomtemperature continuous-wave operation of Mid-IR QCLs have been achieved in the $\sim 3-14 \mu \mathrm{m}$ wavelength range. However, due to the challenges on building up enough optical gain in long-wavelength emission devices above cryogenic temperatures, ${ }^{3} \mathrm{THz}$ QCLs with wavelengths covering from 60 to $300 \mu \mathrm{m}$ are still operated below room temperatures. The best temperature performance has been obtained at $\sim 200 \mathrm{~K}$ using a resonant-phonon (RP) design. ${ }^{4}$ Further improvement on the existing designs requires a better understanding of effects of the electron transport on optical properties, e.g., optical gain, of THz QCLs.

Up to now, several useful theoretical models, e.g., Monte Carlo, ${ }^{5,6}$ nonequilibrium Green's function, ${ }^{7-10}$ and simplified density-matrix ${ }^{11-14}$ models, have been developed to predict the optical properties and electron transports of THz QCLs. Although Monte Carlo and nonequilibrium Green's functions analyses show good agreement between theories and experiments in some aspects, implementations of these two models are difficult, requiring intensive numerical computations. Alternatively, the simplified density matrix model is simple in the analysis and requires much less computation load, while still capturing the essentials of coherent effects such as electron resonant tunneling (RT) transport. It has been shown as one of the most promising candidates for the study of THz QCLs. This model is in essence a set of rate equations but includes electron distributions and coherent dynamics in different subbands. Electrons in each subband are assumed to behave the same, regardless of their kinetic energies. Therefore, this model describes the optical properties and electron transport from a macroscopic point of view, while the microscopic phenomena, e.g., the electron dynamics in the in-plane $k$ space are neglected. Moreover, the present experiments and theoretical predictions have shown the limitations of this macroscopic model. The gain peak frequency calculated by the macroscopic model is overestimated compared to the experimentally measured lasing frequency. ${ }^{4}$ Although coherence effects of RT transport can be described in the macroscopic simplified density matrix model, ${ }^{11}$ another aspect that needs to be considered for a more accurate calculation of optical properties and electron transport is the electron-electron Coulomb interaction, which can induce the renormalizations of the bandstructure (subband energy level) and the Rabi frequency (field).

Direct numerical treatment of many-body Coulomb interaction is complex and hence is often handled at the level of the Hartree-Fock approximation. ${ }^{16}$ In this case, the set of motion equations, in terms of the diagonal and off-diagonal elements of the reduced single-particle density matrix, are well-known as the Hartree-Fock semiconductor Bloch equations, which treat Coulomb effects via bandgap and field renormalization. ${ }^{17}$ For Mid-IR QCLs, the effects of many-body Coulomb interactions on the optical properties and electron transports were considered but the role of coherence of RT transport was neglected. ${ }^{18}$ For THz QCLs, many-body effects on population dynamics were investigated, ${ }^{19}$ but only the injection coupling was considered while the extraction coupling was neglected. Dupont et al. ${ }^{12}$ have demonstrated the importance of extraction coupling on optical properties according to the macroscopic simplified density matrix model but without considering the microscopic properties such as the 
many-body Coulomb interactions. Since many-body Coulomb interactions result in bandstructure renormalization, Coulomb enhancement of the optical transitions, and Coulomb-induced subband coupling, they are expected to play an important role in THz QCLs. However, the effects of electron-electron Coulomb interactions on optical gain of THz QCLs have not yet been reported. In addition, the subband dispersion with different subband effective masses (commonly referred to as nonparabolicity) is known to have a significant effect on the optical properties of laser systems. Thus, the effect of the nonparabolicity induced modifications of subband electrons also needs to be considered. The purpose of this paper is to study the dependence of the intersubband gain spectrum on the electron-electron Coulomb interactions, nonparabolicity, RT, and laser device parameters. The device parameters include external electrical bias, injection- and extraction-coupling strength, dephasing rate, doping density, and temperature.

In this paper, we extend the simplified density matrix model to include the many-body interactions derived from the electron Hamiltonian in the second quantization. It not only takes into account coherent effects in the electron transport through injector and extractor barriers by RT but also distributions of kinetic energy of electrons and many-body effects based on intersubband semiconductor-Bloch equations. The nonparabolicity effect is also approximately considered. The results show that the gain peak frequency calculated by the proposed microscopic density matrix model has a better agreement with the experimentally measured lasing frequency, compared with that calculated from the existing macroscopic density matrix model. The proposed model provides a comprehensive picture of optical properties of THz QCLs, not only enhancing our in-depth understanding of optical gain, but also enabling an accurate prediction of the device performance. More importantly, this model has a low computational load which can greatly simplify the optimization process of active region designs of THz QCLs compared to other full quantum mechanical models. The paper is organized as follows. In Sec. II, we present the intersubband semiconductor-Bloch equations based on the Hartree-Fock approximation, which describes the subband dispersion, Coulomb interaction, and RT effects. In Sec. III, the dependence of subband dispersion, Coulomb interaction and RT transport on the optical gain of THz QCLs are numerically analyzed. The effects of external bias, injectionand extraction-coupling strength, dephasing rate, doping density, and temperature on optical gain are systematically investigated. Finally, the last section summarizes the paper.

\section{EQUATIONS OF MOTION}

Currently, the highest temperature operation of $\mathrm{THz}$ QCL is achieved by using RT injection scheme with three quantum wells in each period. ${ }^{4}$ This record temperature performance is achieved based on a diagonal design; we consider the same design in this paper. Figure 1 shows the conduction band diagram and magnitude squared envelope wave functions of this design in a "tight-binding" scheme, where the injector barrier and extractor barrier are made sufficiently thick to allow one period to be separated into two regions, ${ }^{11,12}$ i.e., the active region and the injector region (see Fig. 1). The energy states

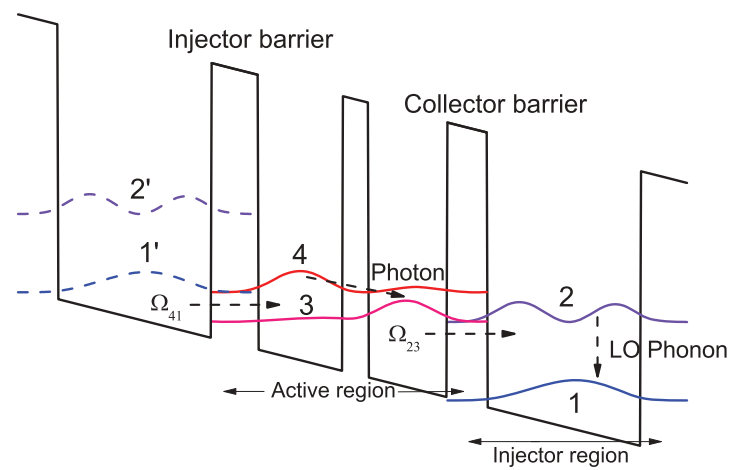

FIG. 1. (Color online) Conduction band diagram and magnitude squared envelope wave functions of a four-level resonant-phonon THz QCL with a diagonal design in the "tight-binding" scheme. The external electric field is $12.3 \mathrm{kV} / \mathrm{cm}$. The radiative transition is from $4 \rightarrow 3$, and depopulation of the lower-laser level is via $3 \rightarrow 2$ (RT) and $(2 \rightarrow 1)$ longitudinal optical phonon scattering. The thickness in angstrom of each layer is given as 49/88/27/82/42/160 starting from the injector barrier. The barriers are indicated in bold fonts. The widest well is doped at $3 \times 10^{10} \mathrm{~cm}^{-2}$.

within either the active region or the injector region are coupled by scattering processes, but energy states from different regions are coupled by tunneling. This localized wave function analysis defined by each region allows us to investigate the effects of RT on the electron transport and gain spectrum.

Assuming single mode operation in THz QCL, the laser field can be written as

$$
E(z, t)=\frac{1}{2} \xi(z, t) e^{i\left[k z-\omega_{\lambda} t-\phi(z)\right]}+\text { c.c. },
$$

where $\xi$ is the slowly varying complex electric field amplitude, $\omega_{\lambda}$ is the laser frequency, $k$ is wave vector, and $\phi$ is the phase.

In order to conveniently treat the many-body problem, we derive the dynamical equations of motion in the second quantized representation. The Hamiltonian of the system can be divided into four parts

$$
H=H_{\mathrm{el}}+H_{\mathrm{rt}}+H_{0}+H_{\text {Coul }} \text {. }
$$

The first two terms

$$
\begin{aligned}
H_{\mathrm{el}}+H_{\mathrm{rt}}= & -\sum_{\mathbf{k}}\left(\mu E b_{4, \mathbf{k}}^{\dagger} b_{3, \mathbf{k}}+c . c\right) \\
& -\sum_{\mathbf{k}}\left[\left(\Delta_{41^{\prime}} / 2\right) b_{1^{\prime}, \mathbf{k}}^{\dagger} b_{4, \mathbf{k}}+c . c\right] \\
& -\sum_{\mathbf{k}}\left[\left(\Delta_{23} / 2\right) b_{3, \mathbf{k}}^{\dagger} b_{2, \mathbf{k}}+c . c\right]
\end{aligned}
$$

are the Hamiltonian for the electron-light coupling and the tunneling effects, respectively. The RT terms are written by a close analogy with the electron-light term, which is similar to the density matrix model firstly proposed by Kazarinov et $a l^{20} b_{j, \mathbf{k}}^{\dagger}\left(b_{j, \mathbf{k}}\right)$ is the creation (annihilation) operator of the electron state in subband $j . \mu$ is the electron charge times the dipole matrix element of laser transition. $\Delta_{41}^{\prime}$ and $\Delta_{23}$ are the injection- and extraction-coupling strengths, which can be derived by a simple tight-binding approach. ${ }^{21}$ The parasitic coupling between levels 1 and 3 as well as levels 2 and 4 is neglected, which is reasonably good for the diagonal design with a large diagonality used in this paper. For the vertical design, 
these two parasitic couplings should be taken into account, and they can be easily treated by analogy with the Eq. (3).

The last two terms ${ }^{22}$

$$
\begin{aligned}
H_{0}+H_{\text {Coul }}= & \sum_{j, \mathbf{k}} \varepsilon_{j, \mathbf{k}} b_{j, \mathbf{k}}^{\dagger} b_{j, \mathbf{k}}+\frac{1}{2} \sum_{u v v^{\prime} u^{\prime}}^{1,2,3,4} \sum_{\mathbf{k} \mathbf{k}^{\prime} \mathbf{q}} V_{\mathbf{q}}^{u v v^{\prime} u^{\prime}} b_{u, \mathbf{k}+\mathbf{q}}^{\dagger} \\
& \times b_{v, \mathbf{k}^{\prime}-\mathbf{q}}^{\dagger} b_{v^{\prime}, \mathbf{k}^{\prime}} b_{u^{\prime}, \mathbf{k}}
\end{aligned}
$$

describe free electrons and electron-electron Coulomb interactions, respectively. $\varepsilon_{j, \mathbf{k}}$ is the $j$ th subband energy, $\mathbf{k}$ is the in-plane wave vector, the $V_{\mathbf{q}}^{u v v^{\prime} u^{\prime}}$ is the screening Coulomb matrix element which reads (in MKS units)

$$
\begin{aligned}
V_{\mathbf{q}}^{u v v^{\prime} u^{\prime}}= & \frac{e^{2}}{2 A \varepsilon_{0} \varepsilon_{r} \varepsilon(q) q} \\
& \int d z \int \varphi_{u}(z) \varphi_{u^{\prime}}(z) e^{-q\left|z-z^{\prime}\right|} \varphi_{v}\left(z^{\prime}\right) \varphi_{v^{\prime}}\left(z^{\prime}\right) d z^{\prime},
\end{aligned}
$$

where $A$ is quantum well area, $\varepsilon_{r}$ is background dielectric constant, and $q=\left|\mathbf{k}-\mathbf{k}^{\prime}\right|$. The near-resonant screening is approximately taken into account via the dielectric function $\varepsilon(q)$ calculated by the single subband screening model. ${ }^{16,23}$ The Coulomb matrix elements are difficult to evaluate numerically, but they can be simplified according to the approaches proposed in Refs. 24 and 25 without loss of high accuracy.

The equations of motion for the polarizations and electron occupation can be derived by using the Heisenberg equations. ${ }^{18,26}$ However, due to the Coulomb interaction terms in Eq. (4), the result is an infinite hierarchy of coupled differential equations. The hierarchy describes the correlation effect in the Coulomb potential. The first-order correlation is induced by the Hartree-Fock contributions, which results in bandstructure and Rabi frequency renormalizations. Scattering and dephasing contributions cause the second-order correlation in the Coulomb potential, and so on. In this paper, we only include the Hartree-Fock contributions and dephasing and scattering contributions at the level of a relaxation-rate approximation. ${ }^{17,18}$ In the rotating-wave approximation, we obtain the following kinetic equations for polarizations $p_{i j, \mathbf{k}}=$ $\left\langle b_{i, \mathbf{k}}^{\dagger} b_{j, \mathbf{k}}\right\rangle \exp \left(-\omega_{\lambda} t\right)$ and electron occupation $n_{i, \mathbf{k}}=\left\langle b_{i, \mathbf{k}}^{\dagger} b_{i, \mathbf{k}}\right\rangle$, where the bracket $\langle\cdots\rangle$ indicates an expectation value

$$
\begin{aligned}
& \frac{d p_{34, \mathbf{k}}}{d t}=-\gamma_{34 p} p_{34, \mathbf{k}}-i\left(\frac{\tilde{\varepsilon}_{43, \mathbf{k}}}{\hbar}-\omega_{\lambda}\right) p_{34, \mathbf{k}}-i \tilde{\Omega}_{0}\left(n_{4, \mathbf{k}}-n_{3, \mathbf{k}}\right)+i \tilde{\Omega}_{41^{\prime}}^{\dagger} p_{31^{\prime}, \mathbf{k}}-i \tilde{\Omega}_{23}^{\dagger} p_{24, \mathbf{k}} \\
& \frac{d p_{41^{\prime}, \mathbf{k}}}{d t}=-\gamma_{41^{\prime} p} p_{41^{\prime}, \mathbf{k}}-i \frac{\tilde{\varepsilon}_{1^{\prime}, \mathbf{k}}}{\hbar} p_{41^{\prime}, \mathbf{k}}-i \tilde{\Omega}_{41^{\prime}}\left(n_{1, \mathbf{k}}-n_{4, \mathbf{k}}\right)-i \tilde{\Omega}_{0}^{\dagger} p_{31^{\prime}, \mathbf{k}} \\
& \frac{d p_{23, \mathbf{k}}}{d t}=-\gamma_{23 p} p_{23, \mathbf{k}}-i \frac{\tilde{\varepsilon}_{32, \mathbf{k}}}{\hbar} p_{23, \mathbf{k}}-i \tilde{\Omega}_{23}\left(n_{3, \mathbf{k}}-n_{2, \mathbf{k}}\right)+i \tilde{\Omega}_{0}^{\dagger} p_{24, \mathbf{k}} \\
& \frac{d p_{31^{\prime}, \mathbf{k}}}{d t}=-\gamma_{31^{\prime} p} p_{31^{\prime}, \mathbf{k}}-i\left(\frac{\tilde{\varepsilon}_{1^{\prime} 3, \mathbf{k}}}{\hbar}-\omega_{\lambda}\right) p_{31^{\prime}, \mathbf{k}}-i \tilde{\Omega}_{0}^{\dagger} p_{41^{\prime}, \mathbf{k}}+i \tilde{\Omega}_{41^{\prime}}^{\dagger} p_{34, \mathbf{k}}-i \tilde{\Omega}_{23}^{\dagger} p_{21^{\prime}, \mathbf{k}} \\
& \frac{d p_{24, \mathbf{k}}}{d t}=-\gamma_{24 p} p_{24, \mathbf{k}}-i\left(\frac{\tilde{\varepsilon}_{42, \mathbf{k}}}{\hbar}-\omega_{\lambda}\right) p_{24, \mathbf{k}}+i \tilde{\Omega}_{0}^{\dagger} p_{23, \mathbf{k}}+i \tilde{\Omega}_{41^{\prime}}^{\dagger} p_{21^{\prime}, \mathbf{k}}-i \tilde{\Omega}_{23}^{\dagger} p_{34, \mathbf{k}} \\
& \frac{d p_{21^{\prime}, \mathbf{k}}}{d t}=-\gamma_{21^{\prime} p} p_{21^{\prime}, \mathbf{k}}-i\left(\frac{\tilde{\varepsilon}_{1^{\prime}, \mathbf{k}}}{\hbar}-\omega_{\lambda}\right) p_{21^{\prime}, \mathbf{k}}+i \tilde{\Omega}_{41^{\prime}}^{\dagger} p_{24, \mathbf{k}}-i \tilde{\Omega}_{23}^{\dagger} p_{31^{\prime}, \mathbf{k}} \\
& \frac{d n_{4, \mathbf{k}}}{d t}=-i\left(\tilde{\Omega}_{0}^{\dagger} p_{34, \mathbf{k}}-\tilde{\Omega}_{0} p_{34, \mathbf{k}}^{\dagger}\right)-i\left(\tilde{\Omega}_{41^{\prime}} p_{41^{\prime}, \mathbf{k}}^{\dagger}-\tilde{\Omega}_{41^{\prime}}^{\dagger} p_{41^{\prime}, \mathbf{k}}\right)-\gamma_{4}\left[n_{4, \mathbf{k}}-f_{4, \mathbf{k}}\left(\mu_{4, e}, T_{4, e}\right)\right]-\gamma_{43}\left[n_{4, \mathbf{k}}-f_{4, \mathbf{k}}\left(\mu_{43}, T_{l}\right)\right]-\gamma_{\mathrm{sp}} n_{4, \mathbf{k}} \\
& \frac{d n_{3, \mathbf{k}}}{d t}=-i\left(\tilde{\Omega}_{0} p_{34, \mathbf{k}}^{\dagger}-\tilde{\Omega}_{0}^{\dagger} p_{34, \mathbf{k}}\right)-i\left(\tilde{\Omega}_{23}^{\dagger} p_{23, \mathbf{k}}-\tilde{\Omega}_{23} p_{23, \mathbf{k}}^{\dagger}\right)-\gamma_{3}\left[n_{3, \mathbf{k}}-f_{3, \mathbf{k}}\left(\mu_{3, e}, T_{3, e}\right)\right]-\gamma_{43}\left[n_{3, \mathbf{k}}-f_{3, \mathbf{k}}\left(\mu_{43}, T_{l}\right)\right]+\gamma_{\mathrm{sp}} n_{4, \mathbf{k}} \\
& \frac{d n_{2, \mathbf{k}}}{d t}=-i\left(\tilde{\Omega}_{23} p_{23, \mathbf{k}}^{\dagger}-\tilde{\Omega}_{23}^{\dagger} p_{23, \mathbf{k}}\right)-\gamma_{2}\left[n_{2, \mathbf{k}}-f_{2, \mathbf{k}}\left(\mu_{2, e}, T_{2, e}\right)\right]-\gamma_{21^{\prime}}\left[n_{2, \mathbf{k}}-f_{2, \mathbf{k}}\left(\mu_{21^{\prime}}, T_{l}\right)\right] \\
& \frac{d n_{1^{\prime}, \mathbf{k}}}{d t}=-i\left(\tilde{\Omega}_{41^{\prime}}^{\dagger} p_{41^{\prime}, \mathbf{k}}-\tilde{\Omega}_{41^{\prime}} p_{41^{\prime}, \mathbf{k}}^{\dagger}\right)-\gamma_{1^{\prime}}\left[n_{1^{\prime}, \mathbf{k}}-f_{1^{\prime}, \mathbf{k}}\left(\mu_{1^{\prime}, e}, T_{1, e}\right)\right]-\gamma_{21^{\prime}}\left[n_{1^{\prime}, \mathbf{k}}-f_{1^{\prime}, \mathbf{k}}\left(\mu_{21^{\prime}}, T_{l}\right)\right],
\end{aligned}
$$

where

$$
\begin{gathered}
\tilde{\varepsilon}_{u v, \mathbf{k}}=\varepsilon_{u, \mathbf{k}}-\varepsilon_{v, \mathbf{k}}-\sum_{\mathbf{k}^{\prime} \neq \mathbf{k}}\left(V_{\mathbf{k}-\mathbf{k}^{\prime}}^{u u u} n_{u, \mathbf{k}^{\prime}}-V_{\mathbf{k}-\mathbf{k}^{\prime}}^{v v v v} n_{v, \mathbf{k}^{\prime}}\right)+\sum_{\mathbf{k}^{\prime} \neq \mathbf{k}}\left(n_{u, \mathbf{k}^{\prime}}-n_{v, \mathbf{k}^{\prime}}\right) V_{\mathbf{k}-\mathbf{k}^{\prime}}^{u v u v} \\
\tilde{\Omega}_{0}=\frac{\mu \xi}{2 \hbar}+\frac{1}{\hbar} \sum_{\mathbf{k}^{\prime} \neq \mathbf{k}} V_{\mathbf{k}-\mathbf{k}^{\prime}}^{4334} p_{43, \mathbf{k}^{\prime}}-\frac{2}{\hbar} V_{0}^{4343} \sum_{\mathbf{k}^{\prime}} p_{43, \mathbf{k}^{\prime}} \\
\tilde{\Omega}_{u v}=\frac{\Delta_{u v}}{2 \hbar}+\frac{1}{\hbar} \sum_{\mathbf{k}^{\prime} \neq \mathbf{k}} V_{\mathbf{k}-\mathbf{k}^{\prime}}^{u v v u} p_{u v, \mathbf{k}^{\prime}}-\frac{2}{\hbar} V_{0}^{u v v v} \sum_{\mathbf{k}^{\prime}} p_{u v, \mathbf{k}^{\prime}}
\end{gathered}
$$



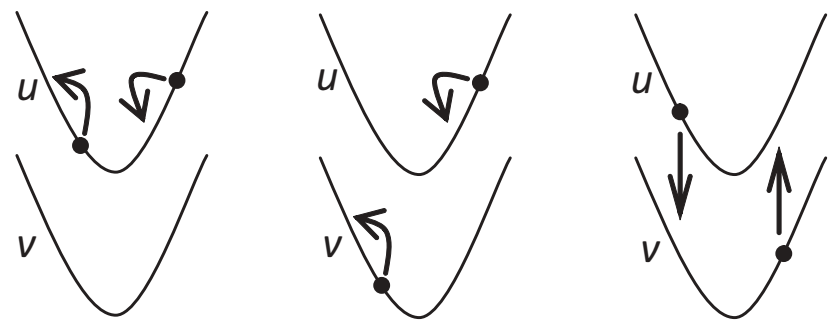

FIG. 2. Graphic representations of the Coulomb interactions between the subbands $u$ and $v$ (from left to right): exchange self-energy, excitonic enhancement, and depolarization.

where $\gamma_{i j p}$ is the dephasing rate associated with energy levels $i$ and $j \cdot \gamma_{j}$ is the intrasubband electron-electron scattering rate at level $j, \gamma_{i j}$ is the combined electron-electron and electronphonon scattering rate between levels $i$ and $j$. The lifetime of the upper-laser level as a function of temperature is approximated in the form of $\gamma_{43}=\gamma_{430} \exp \left[\hbar\left(\omega_{\mathrm{LO}}-\omega_{\lambda}\right) / k_{b} T_{4 e}\right]$, where $\gamma_{430}$ is the raw longitudinal optical (LO) phononscattering rate when the upper-state electrons can sufficiently emit LO phonons and $\omega_{\mathrm{LO}}$ is the energy of LO phonon. $\gamma_{\mathrm{sp}}$ is the spontaneous emission rate. $T_{j, e}$ is the electron temperature at level $j$, and $T_{l}$ is the lattice temperature. $f_{j, \mathbf{k}}$ is the Fermi-Dirac distribution with chemical potential $\mu_{j, e}$ at level $j$. The chemical potentials and temperatures are determined by electron number conservation and energy conservation. ${ }^{17}$ The terms with coefficients $V_{\mathbf{k}-\mathbf{k}^{\prime}}^{\text {uuu }}$ refer to the exchange self-energy, $V_{\mathbf{k}-\mathbf{k}^{\prime}}^{u v v u}$ to the excitonic enhancement, and $V_{0}^{u v u v}$ to the depolarization. Processes corresponding to these contributions are shown in Fig. 2. The influence of the subband dispersion, namely the nonparabolicity, is represented by using the effective mass of electrons $m^{*}$. For subband $j$, we have $\varepsilon_{j, \mathbf{k}}=\varepsilon_{j}+\hbar^{2} \mathbf{k}^{2} / 2 m_{j}^{*}$. For our structure, the calculation shows that $m_{1}^{*} \approx 0.0670 m_{0}, m_{2}^{*} \approx m_{3}^{*} \approx 0.0723 m_{0}$, and $m_{4}^{*} \approx$ $0.0743 m_{0}\left(m_{0}\right.$ is the free electron mass) according to the Ekenberg's model. ${ }^{27}$

In the previous equations of motion, the relaxation-rate approximation approach is employed to calculate dephasing and scattering contributions, ${ }^{17,18,28}$ because a full kinetic treatment of dephasing and scattering terms based on the Boltzmann equation requires extremely long computational time. In this relaxation-rate approximation, the dephasing contributions on the polarizations is treated as

$$
\frac{d p_{i j, \mathbf{k}}}{d t}=-\gamma_{i j p} p_{i j, \mathbf{k}},
$$

where $\gamma_{i j p}$ denotes an effective dephasing rate.

As for the scattering contributions, the influence of scattering on the electron distributions is treated as the relaxation of a given population distribution $n_{u, \mathbf{k}}$ to a quasi-equilibrium Fermi-Dirac distribution $f_{u, \mathbf{k}}\left(T_{i}, \mu_{i}\right)$ with temperature $T_{i}$ and chemical potential $\mu_{i}$ as $\gamma_{u}\left(n_{u, \mathbf{k}}-f_{u, \mathbf{k}}\right)$ at energy level $i$. Two main scattering contributions considered in THz QCLs are electron-electron scattering and electron-phonon scattering.

For the intrasubband scattering, we only consider the electron-electron scattering due to the much smaller intrasubband electron-phonon scattering rate. The electron distribution of each subband (e.g., subband $u$ ) is relaxed to a Fermi-Dirac distribution by this intrasubband scattering at the correspond- ing electron temperature $T_{u, e}$ and chemical potential $\mu_{u, e}$. The actual value of electron temperature and chemical potential can be determined by the conditions of particle and energy conservation

$$
\begin{aligned}
\sum_{\mathbf{k}} n_{u, \mathbf{k}} & =\sum_{\mathbf{k}} f_{u, \mathbf{k}}\left(\mu_{u, e}, T_{u, e}\right) \\
\sum_{\mathbf{k}} \varepsilon_{u, \mathbf{k}} n_{u, \mathbf{k}} & =\sum_{\mathbf{k}} \varepsilon_{u, \mathbf{k}} f_{u, \mathbf{k}}\left(\mu_{u, e}, T_{u, e}\right) .
\end{aligned}
$$

As for the intersubband scattering, two contributions are considered. First, for the intersubband electron-phonon scattering between levels $u$ and $v$, energy is dissipated from the electrons to the lattice. In this case, electrons of these two energy levels relax to a shared Fermi-Dirac distribution with lattice temperature $T_{l}$ and chemical potential $\mu_{u v, l}$. The chemical potential can be determined by the particle conservation

$$
\sum_{\mathbf{k}} \sum_{i=u, v} n_{i, \mathbf{k}}=\sum_{\mathbf{k}} \sum_{i=u, v} f_{i, \mathbf{k}}\left(\mu_{u v, l}, T_{l}\right) .
$$

Second, in the case of the intersubband electron-electron scattering between levels $u$ and $v$, electrons of these two energy levels relax to a Fermi-Dirac distribution with electron temperature $T_{u v, e}$ and chemical potential $\mu_{u v, e}$. According to the particle and energy conservation, it has

$$
\begin{aligned}
\sum_{\mathbf{k}} \sum_{i=u, v} n_{i, \mathbf{k}} & =\sum_{\mathbf{k}} \sum_{i=u, v} f_{i, \mathbf{k}}\left(\mu_{u v, e}, T_{u v, e}\right) \\
\sum_{\mathbf{k}} \sum_{i=u, v} \varepsilon_{i, \mathbf{k}} n_{i, \mathbf{k}} & =\sum_{\mathbf{k}} \sum_{i=u, v} \varepsilon_{i, \mathbf{k}} f_{i, \mathbf{k}}\left(\mu_{u v, e}, T_{u v, e}\right) .
\end{aligned}
$$

If the electron-electron and electron-phonon scattering between levels $u$ and $v$ occur on similar timescales, electrons of these two energy levels driven by electron-electron scattering still relax to a Fermi-Dirac distribution with lattice temperature $T_{l}$ and chemical potential $\mu_{u v, l} .{ }^{18}$ Because the electron-electron and electron-phonon scattering between the upper-laser level and the lower-laser level occur on similar timescales for THz QCLs due to thermally activated phonon scattering at our chosen temperatures, electrons of these two energy levels are assumed to share a Fermi-Dirac distribution at the lattice temperature, as shown in Eqs. $(6 \mathrm{~g})$ and (6h). In addition, because the electron-phonon interaction dominates the scattering processes between level 2 and level 1 in $\mathrm{THz}$ QCLs, we neglect the electron-electron scattering between level 2 and 1, as shown in Eqs. (6i) and (6j).

The dynamic equations of motion are solved numerically in the steady state for a small laser field. Using the semiclassical laser theory, the intensity gain $G$ is given by

$$
G=-\frac{2 \omega_{\lambda}}{\varepsilon_{0} n c V_{m} \xi} \operatorname{Im}\left(\sum_{\mathbf{k}} \mu p_{34, \mathbf{k}}\right),
$$

where $\varepsilon_{0}$ is the vacuum permittivity, $n$ is the refractive index, $c$ is the light speed in vacuum, and $V_{m}$ is the volume of one period of active region. 


\section{RESULTS AND DISCUSSIONS}

\section{A. Effects of many-body interaction and nonparabolicity on optical gain}

The results presented here are obtained by numerically solving the equations of motion [Eqs. (6a)-(6m)] for a small laser field (linear absorption). In our calculation, $250 k$ points within each subband are taken. Figure 3 shows the computed gain spectra at resonance based on the design shown in Fig. 1. In order to evaluate the importance of Coulomb interaction and nonparabolicity on optical properties for THz QCLs, we first compare the gain spectra calculated from the microscopic model with "many-body + nonparabolicity" (considering both many-body and nonparabolicity effects), microscopic model with "many-body + parabolicity" (considering both
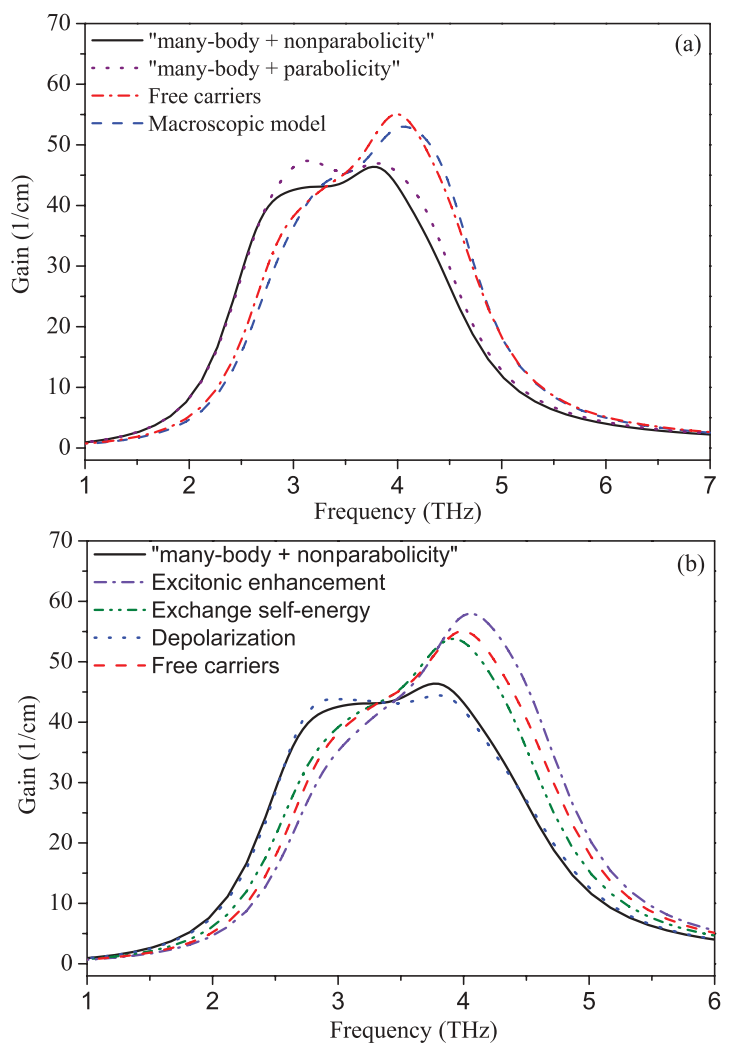

FIG. 3. (Color online) Simulation results for gain spectra at resonance and $100 \mathrm{~K}$. (a) The gain spectra calculated from microscopic model "many-body + nonparabolicity" (solid line), microscopic model "many-body + parabolicity" (dotted lines), microscopic model "free carriers" (dotted-dashed lines), and macroscopic matrix density model (dashed lines). The following default parameters are used: $z_{43}=40.7 \AA, 2 \hbar \Omega_{41}=2.16 \mathrm{meV}$, $2 \hbar \Omega_{23}=4.94 \mathrm{meV}, \gamma_{34 p}=2.4 \times 10^{12} \mathrm{~s}^{-1}, \gamma_{41 p}=4.0 \times 10^{12} \mathrm{~s}^{-1}$, $\gamma_{23 p}=4.5 \times 10^{12} \mathrm{~s}^{-1}, \gamma_{31 p}=3.5 \times 10^{12} \mathrm{~s}^{-1}, \gamma_{24 p}=3.5 \times 10^{12}$ $\mathrm{s}^{-1}, \gamma_{21 p}=3.5 \mathrm{~s}^{-1}, \gamma_{43}=1.3 \times 10^{11} \mathrm{~s}^{-1}, \gamma_{21}=3 \times 10^{12} \mathrm{~s}^{-1}, \gamma_{1}=$ $1.3 \times 10^{12} \mathrm{~s}^{-1}, \gamma_{4}=1.3 \times 10^{12} \mathrm{~s}^{-1}, \gamma_{3}=1.3 \times 10^{12} \mathrm{~s}^{-1}, \gamma_{2}=$ $1.3 \times 10^{12} \mathrm{~s}^{-1}$. The typical values of scattering and dephasing rate are used (Refs. 11 and 19). (b) The interplay of different Coulomb interactions. Solid lines: full many-body effects with nonparabolicity; dashed lines: free carriers; dotted lines: depolarization; dot-dashed lines: excitonic enhancement; dot-dot-dashed lines: exchange self-energy.

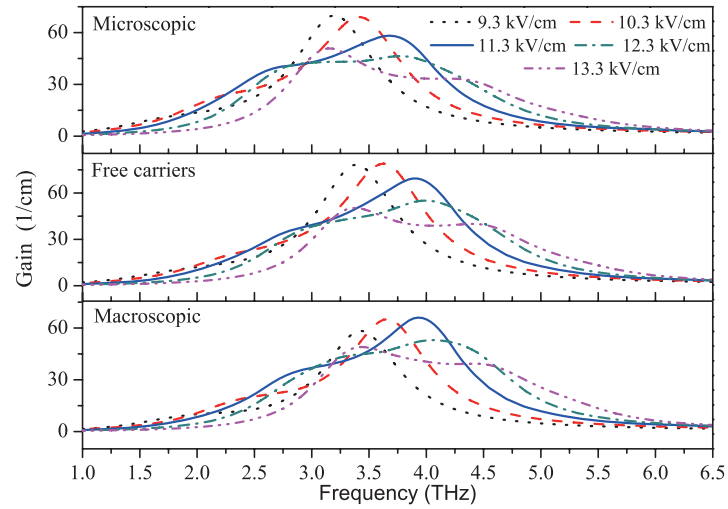

FIG. 4. (Color online) Effects of bias on gain spectra at $100 \mathrm{~K}$. The designed bias is $12.3 \mathrm{kV} / \mathrm{cm}$.

many-body and parabolicity effects), microscopic model with "free carriers" (considering free carriers and nonparabolicity but neglecting the renormalizations of bandstructure and Rabi frequency), and the macroscopic density matrix model (Ref. 12), as shown in Fig. 3(a). Comparing the two gain spectra calculated by the microscopic models with "manybody + nonparabolicity" and "many-body + parabolicity," we found that nonparabolicity causes a slight shift of peak position of gain spectrum to the lower frequency side and reduces the peak gain due to the $k$-space distribution of electrons in subbands with different effective masses. Not only the nonparabolicity but also the many-body Coulomb interactions make the red-shift of gain spectrum and cause the decrease of peak value of optical gain of THz QCLs by comparing the microscopic models with "many-body + nonparabolicity" and "free carriers." The red-shift of the gain spectrum is mainly caused by the depolarization terms with the consideration of the interplay of various many-body interactions, as shown in Fig. 3(b). It is shown that, in our population inverted laser system, the depolarization causes a red-shift of gain spectrum relative to the "free-carrier" model, ${ }^{29}$ but it induces the blue-shift in the usual noninverted absorption system. In addition, exchange self-energy terms renormalize the subband energy level and induce nonparabolicity to slightly red-shift the gain spectrum, and excitonic enhancement terms give a peak near the higher frequency edge of the spectrum and cause the

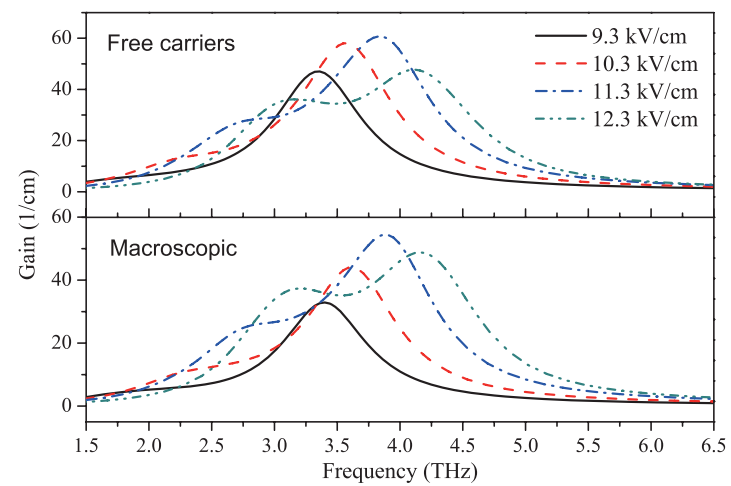

FIG. 5. (Color online) Effects of bias on gain spectra from the free-carrier model and the macroscopic model at $100 \mathrm{~K}$ when the injection-coupling strength is set as $1.2 \mathrm{meV}$. 
slight blue-shift of the gain spectrum in THz QCLs. Therefore, the interplay of various many-body effects leads to the red-shift of gain spectrum. Furthermore, since the macroscopic model does not consider the many-body interactions and nonparabolicity, the obtained peak value and peak frequency are higher as compared to those obtained by the microscopic "many-body + nonparabolicity" model. Moreover, the spectral lineshape, which is important for calculating parameters such as the linewidth enhancement factor, is not accurately predicted by the macroscopic model. Overall, the different spectral features, e.g., different peak values, peak frequency positions, and spectrum lineshapes from two models demonstrate that the microscopic density matrix model can enrich in-depth understanding of optical properties of THz QCLs and enables a more accurate prediction of the gain spectrum.

\section{B. Optical gain spectrum as a function of bias}

Figure 4 shows the gain spectra at different biases calculated from the microscopic model (in subsequent discussions, unless otherwise specified, "microscopic model" refers to the microscopic model "many-body + nonparabolicity"), the free-carrier model, and the macroscopic one. Due to the coherence of RT transport across the injector and extractor barriers, the effects of RT on the broadening mechanism of gain spectrum are complicated for THz QCLs. As shown in Fig. 4, the gain spectrum is broadened as the applied bias is
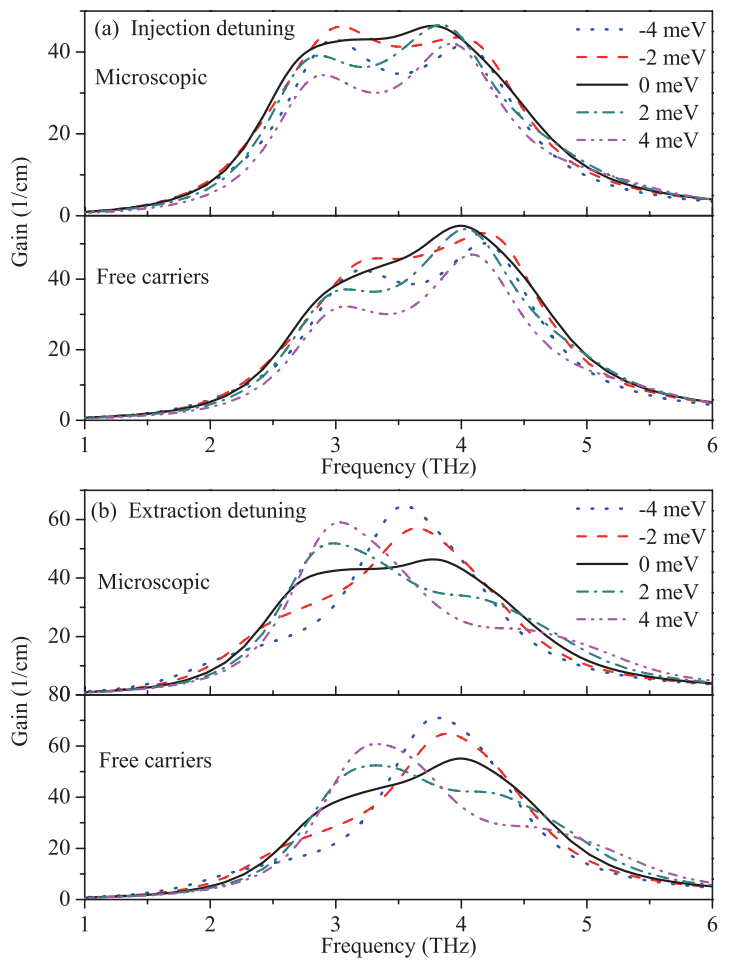

FIG. 6. (Color online) The effects of detunings with and without many-body interactions on the optical gain at $100 \mathrm{~K}$. (a) The gain spectra at different injection detunings $\varepsilon_{14}$ : the extractor level is kept in resonance with the lower-laser level. (b) The gain spectra at different extraction detunings $\varepsilon_{32}$. The injection level is kept to be in resonance with the upper-laser level. close to the designed value. This broadening results from the contributions of the polarization $p_{31, k}$ and $p_{24, k}$ to polarization $p_{34, k}$ due to the indirect $1 \rightarrow 3$ and $4 \rightarrow 2$ radiative transition formed by the coherent coupling $1 \leftrightarrow 4,3 \leftrightarrow 4$, and $3 \leftrightarrow 2$. As the bias increases, levels $1-4$ and 3-2 are close to resonance, then levels $1-3$ and 4-2 become more coherent, hence $1 \rightarrow 3$ and $4 \rightarrow 2$ indirect radiative transitions become stronger and contribute to the spectrum broadening.

It is noted that the gain peak frequency calculated by the microscopic model is lower $(\sim 0.3 \mathrm{THz})$ than that by the macroscopic model, as shown in Fig. 4. Because, as demonstrated in Ref. 4, the macroscopic density matrix model overestimates $(\sim 0.6 \mathrm{THz})$ the gain peak frequency compared to the experimentally measured lasing frequency, therefore the microscopic many-body model discussed in this paper predicts a better result closer to the experimental lasing frequency. However, the gain peak frequency calculated by the microscopic model is still $\sim 0.3 \mathrm{THz}$ higher than the experimental value. This discrepancy is probably caused by the neglected intermodule electron-light scattering in this particular density matrix model. ${ }^{4}$ Due to the choice of basis states from the two isolated modules (the active and the injector modules) in the "tight-binding" scheme (see Fig. 1), this model considers only intramodule scatterings, and hence only one intramodule dipole moment $z_{43}$, but neglects other direct dipole moment contributions, e.g., $z_{42}$. This limit is not inherent to the density matrix model but is due to the choice of the basis states. Further work could be carried out to include this intermodule electron-light scattering.
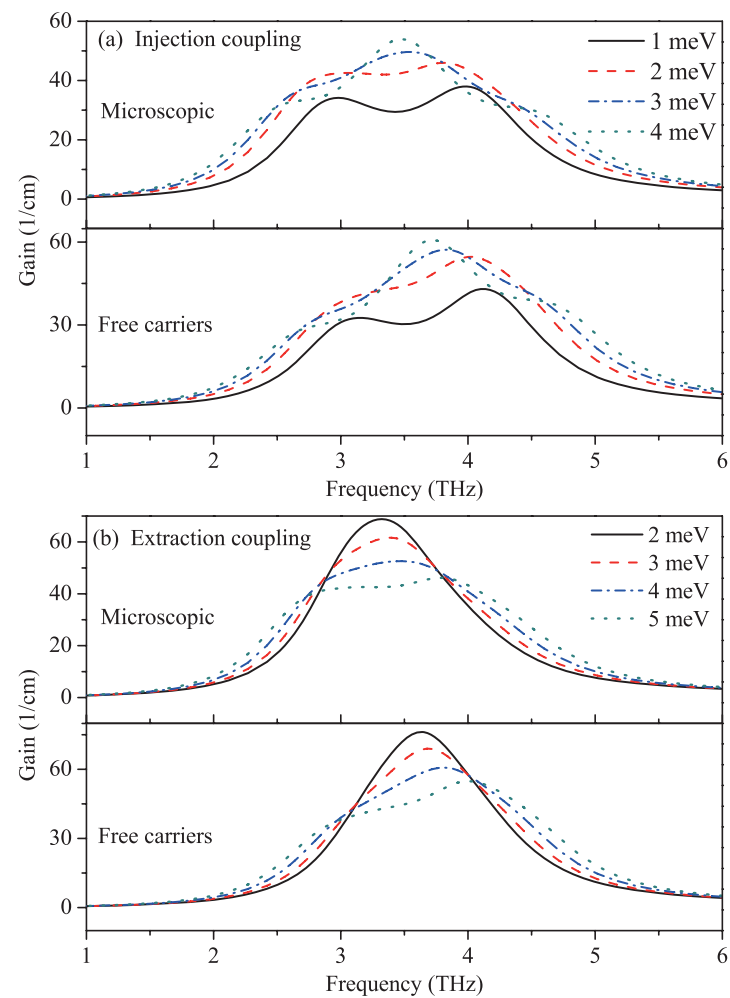

FIG. 7. (Color online) Effects of coupling strength with and without many-body interactions on gain spectra at resonance and $100 \mathrm{~K}$. (a) Gain spectra at different injection-coupling strength. (b) Gain spectra at different extraction-coupling strength. 
In addition, the variation of the gain spectrum with the bias calculated from the microscopic model is different from that calculated from the macroscopic model with the present parameters, as shown in Fig. 4. According to the free-carrier model, the peak gain first slightly increases then decreases as the bias increases before the injection level 1 and upper-laser level 4 are resonantly coupled. In contrast, the peak gain increases as the bias increases in the macroscopic model. Through further calculations, we found that the differences of the bias-dependent spectrum characteristics between the microscopic and macroscopic models depend on the RT parameters, i.e., the coupling strength. As shown in Fig. 5, when the injection-coupling strength is set as $1.2 \mathrm{meV}$, similar variations of gain spectra with the bias between the free-carrier and the macroscopic models are observed, i.e., the peak gain increases as the bias increases before levels 1 and 4 are resonantly coupled. Hence, the bias dependences of gain spectrum in the microscopic many-body and free-carrier models, which is different from that of macroscopic model in Fig. 4, can be attributed to the interplay of many-body interaction, kinetic-energy distribution of electrons and RT effects.

Figure 4 gives the overall effects on the gain spectra when the injection and extraction detunings are simultaneously changed. The respective effects of the injection and extraction detunings on the gain spectra are shown in Fig. 6. The optical gain spectrum changes as the injection and extraction detunings vary due to the energy splitting owing to the 1-4 and 3-2 anticrossing, respectively. By comparing Fig. 6(a) with Fig. 6(b), we found that the peak position and lineshape of gain spectrum strongly follows the variations of extraction detuning. Hence, the extraction detuning is more important than the injection detuning for determining the gain spectrum. In addition, at each injection and extraction detunings, the many-body interactions tend to suppress the high frequency side of gain spectrum and enhance the low frequency side by the comparison of microscopic model and free-carrier one.

\section{Optical gain spectrum as a function of injection- and extraction-coupling strength}

In order to further illustrate the effects of RT on gain spectrum, we simulate the gain spectra at different injectionand extraction-coupling strengths, as shown in Fig. 7. As the injection-coupling strength gradually increases toward the value of extraction coupling strength, the gain spectrum tends to be enhanced, peak frequency follows the variation of coupling strength, and spectrum width is slightly broadened, as shown in Fig. 7(a). When the injection-coupling strength is small enough (smaller than extraction coupling strength), double-peak gain spectrum is generated. Furthermore, by the comparison of the microscopic model and free-carrier one, the many-body interaction tends to suppress the high frequency side of the gain spectrum and enhance the low frequency side.

In contrast, since the extraction coupling strength is larger than the injection-coupling strength, as the extraction coupling strength increases, extraction electron transport tends to be more coherent and the gain spectrum is additionally broadened. In this case, the peak value is reduced owing to the interplay of Coulomb interaction and RT effect due to the indirectly coherent interaction of $p_{24}$. When the extraction coupling strength is large enough (larger than injectioncoupling strength), double-peak gain spectrum is generated. Similarly, owing to modifications to gain spectrum by manybody interactions, the high frequency side of spectrum is suppressed, and the low frequency side is enhanced in the microscopic model, as compared to the free-carrier model.

\section{Optical gain spectrum as a function of dephasing rate}

RT transport is not only influenced by coupling strength but also determined by the dephasing rate. Figure 8(a) shows the effects of the dephasing rate $\gamma_{41} p$ associated with RT transport across the injection barrier on the gain spectrum. Although the decreasing dephasing rate $\gamma_{41 p}$ enhances the gain spectrum, it does not change the lineshape of gain spectrum like the coupling strength. For completeness of analysis, we also give the effects of dephasing rate $\gamma_{34 p}$ associated with laser transition on the gain spectra, as shown in Fig. 8(b). Also, the peak value of gain spectrum decreases, but the lineshape is not changed as the dephasing rate $\gamma_{34 p}$ increases.

\section{E. Optical gain spectrum as a function of doping density and temperature}

Since many-body Coulomb interaction strongly depends on the doping density, we anticipate that doping will strongly affect the gain spectra, as shown in Fig. 9. As the doping density increases, the many-body interactions become stronger,
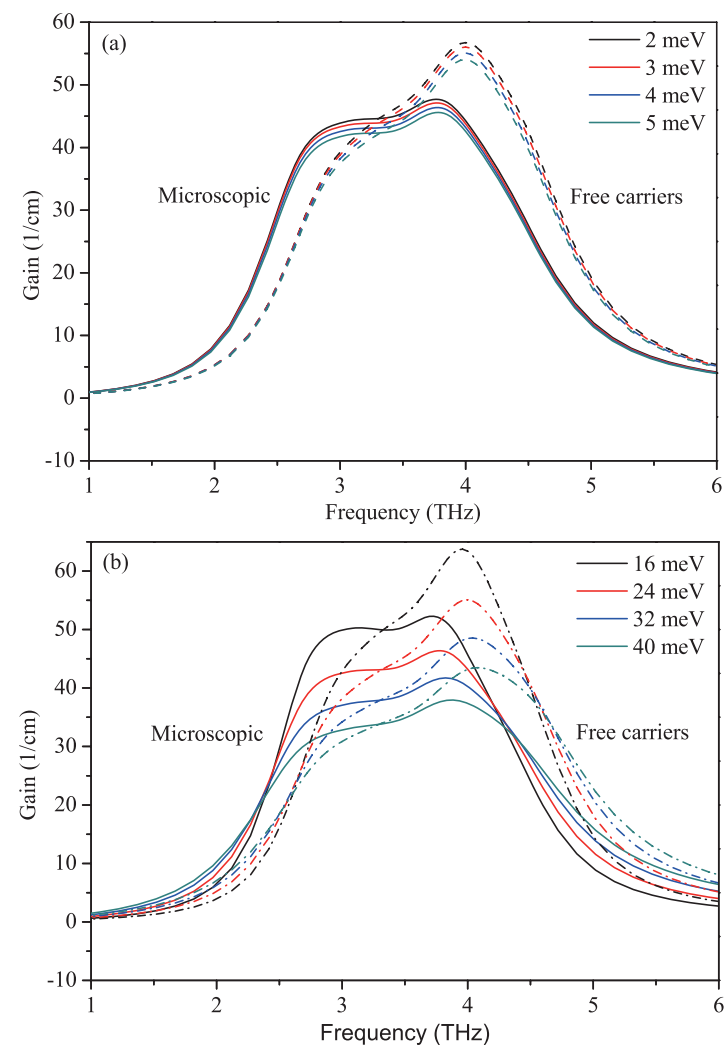

FIG. 8. (Color online) Gain spectra at different dephasing rates $\gamma_{41 p}$ (a) associated with resonant tunneling across the injection barrier, and $\gamma_{34 p}$ (b) associated with laser transition at resonance and $100 \mathrm{~K}$. Solid lines: microscopic model; dashed lines: free carriers. 

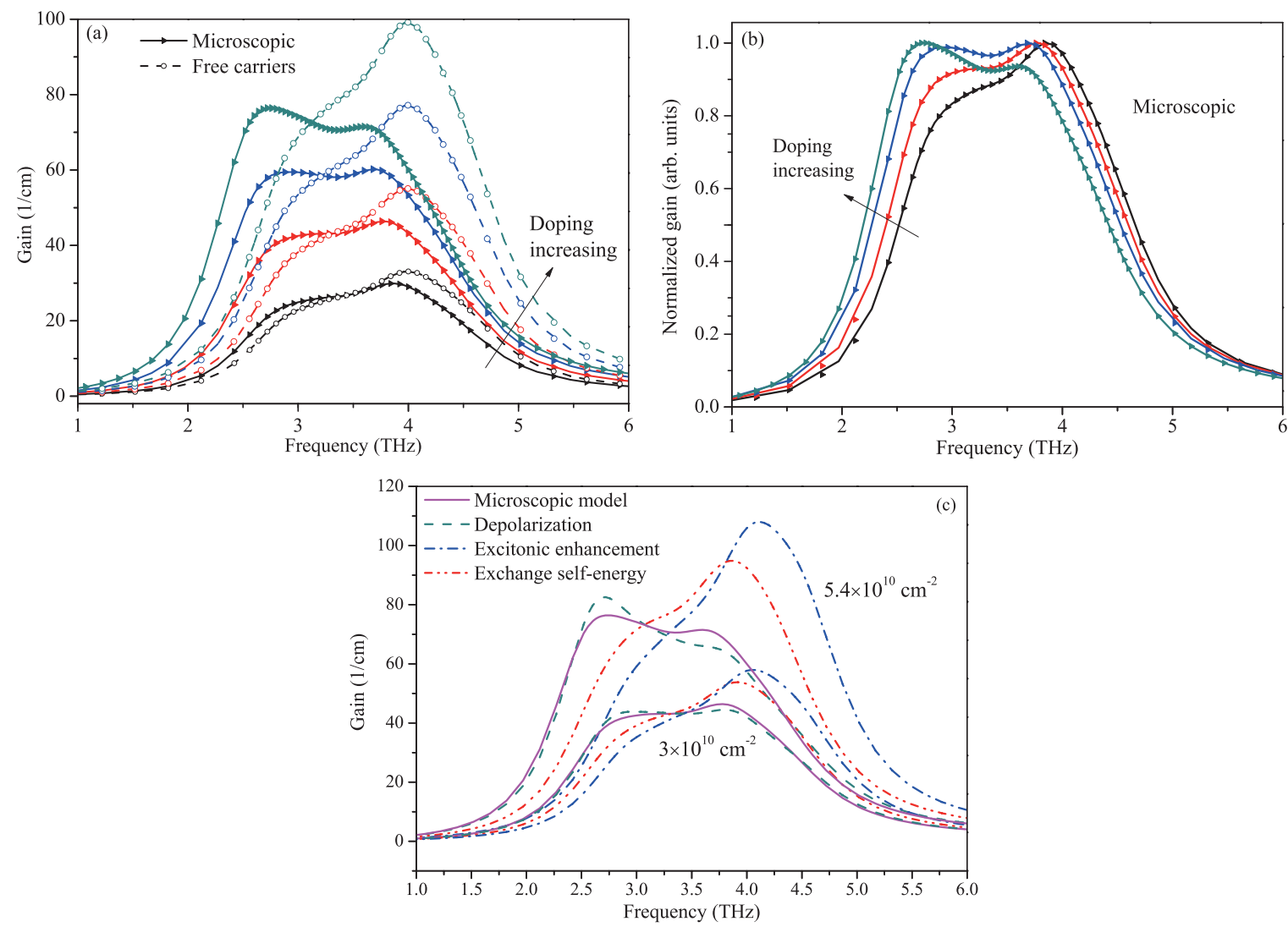

FIG. 9. (Color online) (a) Effects of doping density on gain spectra at resonance and $100 \mathrm{~K}$. The solid lines with right triangle and dashed lines with open circles show the results of microscopic and free-carrier models, respectively. From the bottom to the top for each kind of color line, the doping density is $1.8 \times 10^{10} \mathrm{~cm}^{-2}, 3 \times 10^{10} \mathrm{~cm}^{-2}, 4.2 \times 10^{10} \mathrm{~cm}^{-2}$, and $5.4 \times 10^{10} \mathrm{~cm}^{-2}$. The effects of doping density on lifetimes of energy levels are neglected. (b) Normalized gain spectra from microscopic model at different doping density. (c) The interplay of various Coulomb interactions at doping densities $3 \times 10^{10} \mathrm{~cm}^{-2}$ and $5.4 \times 10^{10} \mathrm{~cm}^{-2}$, respectively. Solid lines: full many-body effects with nonparabolicity; dashed lines: depolarization; dot-dashed lines: excitonic enhancement; dot-dot-dashed lines: exchange self-energy.

and hence the spectra are further red-shifted. Moreover, not only the peak value with doping density is enhanced by many-body interactions, the lineshape of spectrum is modified, as shown in Fig. 9(b). Figure 9(c) shows the interplay of various Coulomb interactions at doping densities of $3 \times 10^{10} \mathrm{~cm}^{-2}$ and $5.4 \times 10^{10} \mathrm{~cm}^{-2}$, respectively. As shown by this figure, the red-shift and spectrum lineshape modification are mainly attributed to the depolarization, as demonstrated in Ref. 30. In contrast, the spectrum lineshape calculated from free-carrier model is not changed with the rise in doping density.

In addition to the previous analysis, temperature is also an important parameter that influences the gain spectrum. The macroscopic model cannot accurately investigate the temperature effects on optical properties of THz QCLs due to the lack of the description of electron distribution in $k$ space. However, temperature effect is important since it can affect the kinetic distribution of electrons. Our results show that the rise in temperature will decrease the peak value of the gain spectrum, as shown in Fig. 10(a). The gain spectrum is slightly blue-shifted, and the lineshape is modified. In addition, according to the results of free carriers, as shown in Fig. 10(b), nonparabolicity induces neglected modifications to the lineshape of the gain spectrum at different temperatures.
Therefore, the spectrum shift and modification of lineshape are induced by the many-body Coulomb interactions.

\section{CONCLUSION}

We have established the Hartree-Fock semiconductor Bloch equations with dephasing and scattering contributions treated at the level of a relaxation-rate approximation, which describes the electron-electron Coulomb interaction, nonparabolicity, and coherence of RT transport. We use the developed model to investigate the optical gain of a four-state RP-based THz QCL. The simulation results calculated from the microscopic model with "many-body + nonparabolicity," the microscopic model with "many-body + parabolicity," the microscopic model with "free carriers," and the macroscopic model are compared to demonstrate the importance of those parameters in the simulation of optical properties of $\mathrm{THz}$ QCLs. The effects of the external bias, injection- and extraction-coupling strength, dephasing rate, doping density, and temperature on optical gain are also systematically investigated. The results show that the gain peak frequency calculated by the microscopic model is closer to the experimentally measured lasing frequency compared with the calculated one 

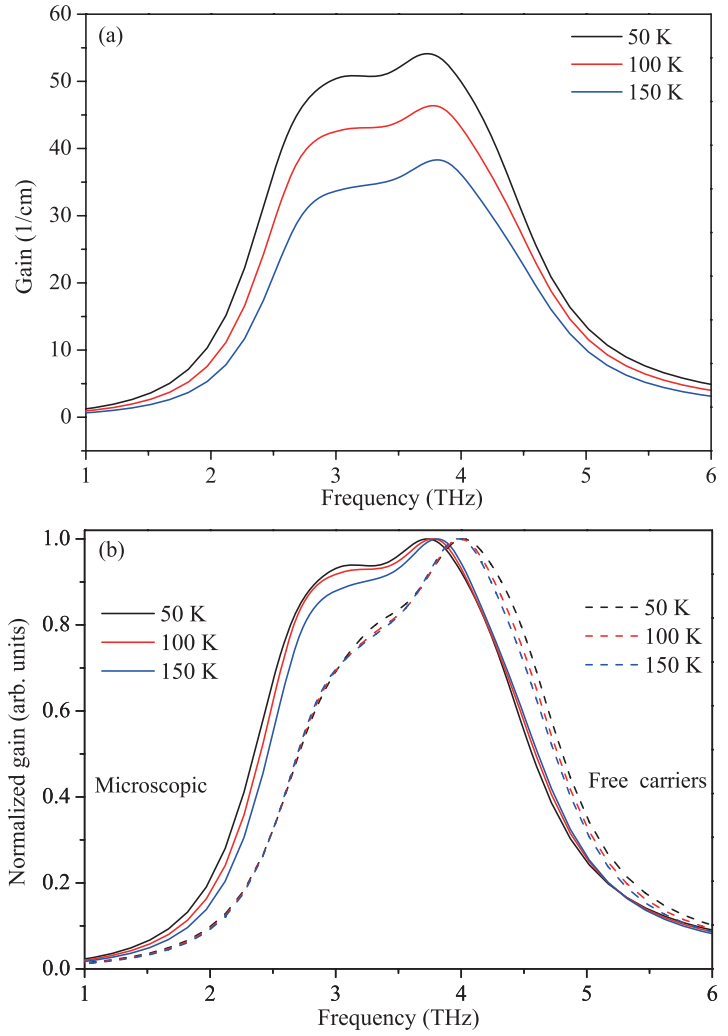

FIG. 10. (Color online) (a) Gain spectra at resonance at different temperatures. (b) Normalized gain spectra at resonance. Solid lines: microscopic model; dashed lines: free-carrier model.

by the macroscopic model. Specifically, both the many-body Coulomb interaction and nonparabolicity cause the red-shift of gain spectrum and reduce the peak gain. The interplay of various many-body interactions reveals that the optical spectral red-shift is mainly caused by the depolarization terms in $\mathrm{THz}$ QCLs, and this red-shift in our population inverted system is contrary to that in the usual noninverted absorption case. Furthermore, the gain spectrum is enhanced and slightly broadened as the injection-coupling strength increases, while an increasing extraction-coupling strength reduces the peak value and broadens the gain spectrum. When the extraction and injection-coupling strengths have different values as to favor one side, the double-peaked gain spectrum is generated. In addition, as the doping density increases, the gain spectrum is red-shifted and modified, while the increasing temperature slightly blue-shifts the gain spectrum.

The microscopic model discussed in this paper provides a relatively comprehensive picture of optical properties of $\mathrm{THz}$ QCLs and enables a more accurate and faster prediction and calculation of the device performance, e.g., gain spectra, current-voltage characteristics, optical output power, and amplitude-phase coupling (linewidth enhancement factor). It also provides an essential design tool for the optimization of the quantum wells/barriers thicknesses to improve the performance of $\mathrm{THz}$ QCLs, such as the maximum operation temperature. There are still certain aspects that the model can be further improved. First, this model takes into account dephasing mechanisms in laser transition and transport coherence but only considers them under the relaxation-rate approximation using phenomenological values, where the nondiagonal scattering contributions are neglected. Nondiagonal scattering contribution is expected to yield not only a smaller linewidth but also a reshaping of the gain spectrum. ${ }^{17} \mathrm{~A}$ more stringent treatment of the dephasing parameters is required for future theoretical studies. Another limitation is that the present implementation of microscopic model considers only intramodule electron-light scatterings but neglects intermodule dipole moment contributions due to the choice of the basis states from the isolated modules in the "tight-binding" scheme. Further study is required to include intermodule electron-light scattering.

\section{ACKNOWLEDGMENTS}

We would like to acknowledge financial support from Nanyang Technological University (NTU) (M58040017), Defence Research and Technology Office, Singapore (TL9009105606-01), and Ministry of Education, Singapore (MOE2011-T2-2-147). Support from the CNRS InternationalNTU-Thales Research Alliance (CINTRA) Laboratory, UMI 3288 , Singapore 637553, is also acknowledged.
*Corresponding author: qjwang@ntu.edu.sg

${ }^{1}$ J. Faist, F. Capasso, D. L. Sivco, C. Sirtori, A. L. Hutchinson, and A. Y. Cho, Science 264, 553 (1994).

${ }^{2}$ R. Köhler, A. Tredicucci, F. Beltram, H. E. Beere, E. H. Linfield, A. G. Davies, D. A. Ritchie, R. C. Iotti, and F. Rossi, Nature 417, 156 (2002).

${ }^{3}$ B. S. Williams, Nat. Photonics 1, 517 (2007).

${ }^{4}$ S. Fathololoumi, E. Dupont, C. W. I. Chan, Z. R. Wasilewski, S. R. Laframboise, D. Ban, A. Mátyás, C. Jirauschek, Q. Hu, and H. C. Liu, Opt. Express 20, 3866 (2012).

${ }^{5}$ C. Jirauschek and P. Lugli, J. Appl. Phys. 105, 123102 (2009).

${ }^{6}$ J. T. Lu and J. C. Cao, Appl. Phys. Lett. 88, 061119 (2006).

${ }^{7}$ T. Liu, T. Kubis, Q. J. Wang, and G. Klimeck, Appl. Phys. Lett. 100, 122110 (2012).
${ }^{8}$ T. Kubis, C. Yeh, P. Vogl, A. Benz, G. Fasching, and C. Deutsch, Phys. Rev. B 79, 195323 (2009)

${ }^{9}$ R. Nelander and A. Wacker, Appl. Phys. Lett. 92, 081102 (2008).

${ }^{10}$ T. Schmielau and M. F. Pereira, Appl. Phys. Lett. 95, 23111 (2009).

${ }^{11}$ S. Kumar and Q. Hu, Phys. Rev. B 80, 245316 (2009).

${ }^{12}$ E. Dupont, S. Fathololoumi, and H. C. Liu, Phys. Rev. B 81, 205311 (2010)

${ }^{13}$ G. Beji, Z. Ikonić, C. A. Evans, D. Indjin, and P. Harrison, J. Appl. Phys. 109, 013111 (2011).

${ }^{14}$ R. Terazzi and J. Faist, New J. Phys. 12, 033045 (2010).

${ }^{15}$ S. Kumar, C. W. I. Chan, Q. Hu, and J. L. Reno, Nat. Physics 7, 166 (2010). 
${ }^{16} \mathrm{H}$. Haug and S. W. Koch, Quantum Theory of the Optical Electronic Properties of Semiconductors (World Scientific Publishing, Singapore, 2009).

${ }^{17}$ W. W. Chow, S. W. Koch, and M. Sargent III, Semiconductor-Laser Physics (Springer-Verlag, Berlin, 1994).

${ }^{18}$ I. Waldmueller, W. W. Chow, E. W. Young, and M. C. Wanke, IEEE J. Quantum Electron. 42, 292 (2006).

${ }^{19}$ F. Wang, X. G. Guo, and J. C. Cao, J. Appl. Phys. 108, 083714 (2010).

${ }^{20}$ R. F. Kazarinov and R. A. Suris, Sov. Phys. Semicond. 6, 120 (1972) [Fiz. Tech. Poluprovodn. 6, 148 (1972)].

${ }^{21}$ G. Bastard, Wave Mechanics Applied to Semiconductor Heterostructures (Les Editions de Physique, Paris, 1988).

${ }^{22}$ D. E. Nikonov, A. Imamoğlu, L. V. Butov, and H. Schmidt, Phys. Rev. Lett. 79, 4633 (1997).
${ }^{23}$ J. T. Lu and J. C. Cao, Appl. Phys. Lett. 89, 211115 (2006).

${ }^{24}$ M. F. Pereira, S.-C. Lee, and A. Wacker, Phys. Rev. B 69, 205310 (2004).

${ }^{25}$ O. Bonno, J.-L. Thobel, and F. Dessenne, J. Appl. Phys. 97, 043702 (2005).

${ }^{26}$ I. Waldmueller, J. Forstner, and A. Knorr, in Nonequilibrium Physics at Short Time Scales, edited by K. Morawetz (Springer, Berlin, 2004).

${ }^{27}$ U. Ekenberg, Phys. Rev. B 40, 7714 (1989).

${ }^{28}$ W. W. Chow, H. C. Schneider, S. W. Koch, C.-H. Chang, L. Chrostowski, and C. J. Chang-Hasnain, IEEE J. Quantum Electron. 38, 402 (2002).

${ }^{29}$ H. C. Liu and A. J. Spring Thorpe, Phys. Rev. B 61, 15629 (2000). ${ }^{30}$ M. V. Kisin, M. A. Stroscio, G. Belenky, and S. Luryi, Appl. Phys. Lett. 73, 2075 (1998). 